\title{
Leak Test of Sensors with the Test Medium compressed Air
}

\author{
Dr. Joachim Lapsien \\ CETA Testsysteme GmbH, Marie-Curie-Str. 35-37, 40721 Hilden, GERMANY, \\ E-Mail: joachim.lapsien@cetatest.com
}

\begin{abstract}
As sensors are used in the diverse industrial applications this component has to fulfill a variety of requirements. This includes leak tightness against dirt and moisture, whose requirements are specified in the IP protection classes. Penetration of liquids can cause serious malfunction of the sensor and the associated electronics. Thus, the sensors have to be leak tight. The leak testing of the sensors as an end-of-line test integrated in the production line is of particular importance. The basics of leak testing and important practical aspects of the leak test process are treated.
\end{abstract}

Key words: leak tightness, IP protection classes, leak test, end-of-line test, hood test.

\section{Test Medium compressed Air}

The definition of the IP protection classes describes how a laboratory test has to be performed. But of course, such a test cannot be integrated as $100 \%$-inline process in the production line. Based on the results of the laboratory experiments the requirements for a corresponding leak test in the production line have to be worked out. Ideally the test pressure and the permissible leak rate can be defined.

Depending on the leak rate a suitable test medium and test process have to be chosen to fulfill the technical requirement. Table 1 shows some of the most common used test media and their specific features. Widespread is the use of the test medium compressed air for the $100 \%$ in-line leak testing in industrial production lines. The test medium compressed air can be used down to an air leak rate of $10^{-3} \mathrm{mbar}{ }^{*} / \mathrm{s}$ (depending on the test part). If a sensor has to be waterproof an air leak rate of $10^{-2} \mathrm{mbar}^{*} / \mathrm{s}$ $\left(=0,6 \mathrm{~cm}^{3} / \mathrm{min}\right)$ is commonly assumed. Typical examples of automotive sensors, which are tested with compressed air are oil pressure sensors, ABS sensors, level sensors.

Tab. 1. Overview about leak test media $\left(1 \mathrm{mbar}^{*} / \mathrm{s}=60 \mathrm{~cm}^{3} / \mathrm{min}\right)$.

\begin{tabular}{|c|c|c|c|}
\hline Test Medium & Air Leak Rate & Method & Remarks \\
\hline Water & $>10^{-2} \mathrm{mbar}^{\star} \mathrm{l} / \mathrm{s}$ & Detection of bubbles & $\begin{array}{l}\text { - Qualitative method } \\
\text { - Localisation of leaks }\end{array}$ \\
\hline Compressed Air & $>10^{-3} \mathrm{mbar}^{*} \mathrm{l} / \mathrm{s}$ & $\begin{array}{l}\text { Measurement of pressure decay } \\
\text { resp. pressure rise (relative } \\
\text { pressure sensor, differential } \\
\text { pressure sensor) }\end{array}$ & $\begin{array}{l}\text { - Quantitative method } \\
\text { - Easy to handle } \\
\text { - Depending on the volume } \\
\text { - Temperature sensitivity } \\
\text { - Integral method } \\
\text { - Localisation of leaks } \\
=>\text { Use of leak spray }\end{array}$ \\
\hline $\begin{array}{l}\text { Hydrogen } \\
\text { (Forming Gas) }\end{array}$ & $>10^{-6} \mathrm{mbar}^{*} \mathrm{l} / \mathrm{s}$ & Measurement of $\mathrm{H}_{2}$-concentration & $\begin{array}{l}\text { - Quantitative method } \\
\text { - No volume dependency } \\
\text { - No temperature sensitivity } \\
\text { - Sniffing: Localisation of leaks }\end{array}$ \\
\hline Helium & $>10^{-9} \mathrm{mbar}^{*} \mathrm{l} / \mathrm{s}$ & Measurement of He-concentration & $\begin{array}{l}\text { - Quantitative method } \\
\text { - No volume dependency } \\
\text { - No temperature sensitivity } \\
\text { - Evacuation for small leak rates } \\
\text { - Sniffing: Localisation of leaks }\end{array}$ \\
\hline
\end{tabular}




\section{Test Method "Pressure Decay Test" for direct fillable Test Parts}

Test parts with small volumes, which can be filled directly, have often to be tested in a very short total test time by the use of compressed air.

The overall test consists of the consecutive phases: filling, stabilizing, testing and dumping. In the filling phase, the test part has to be filled to reach the test pressure. The stabilizing phase is necessary so that air disturbances (caused by the filling process and generated by the switching of the internal valves of the test device) can subside. In case of a positive relative test pressure the air is compressed adiabatically during the filling process, which heats up the air. So, the temperature of the compressed air has to adjust to the temperature of the test part. In the testing phase, the rate of pressure loss is measured and compared with the permitted tolerances. The testing phase must be long enough to generate a significant measurement value. In the testing phase mostly a very sensitive differential pressure sensor is activated to measure the pressure decay with high resolution. A stable measurement phase is characterized by the effect, that the leakageinduced pressure loss is proportional to time. Finally follows the dumping phase (see Fig. 1).

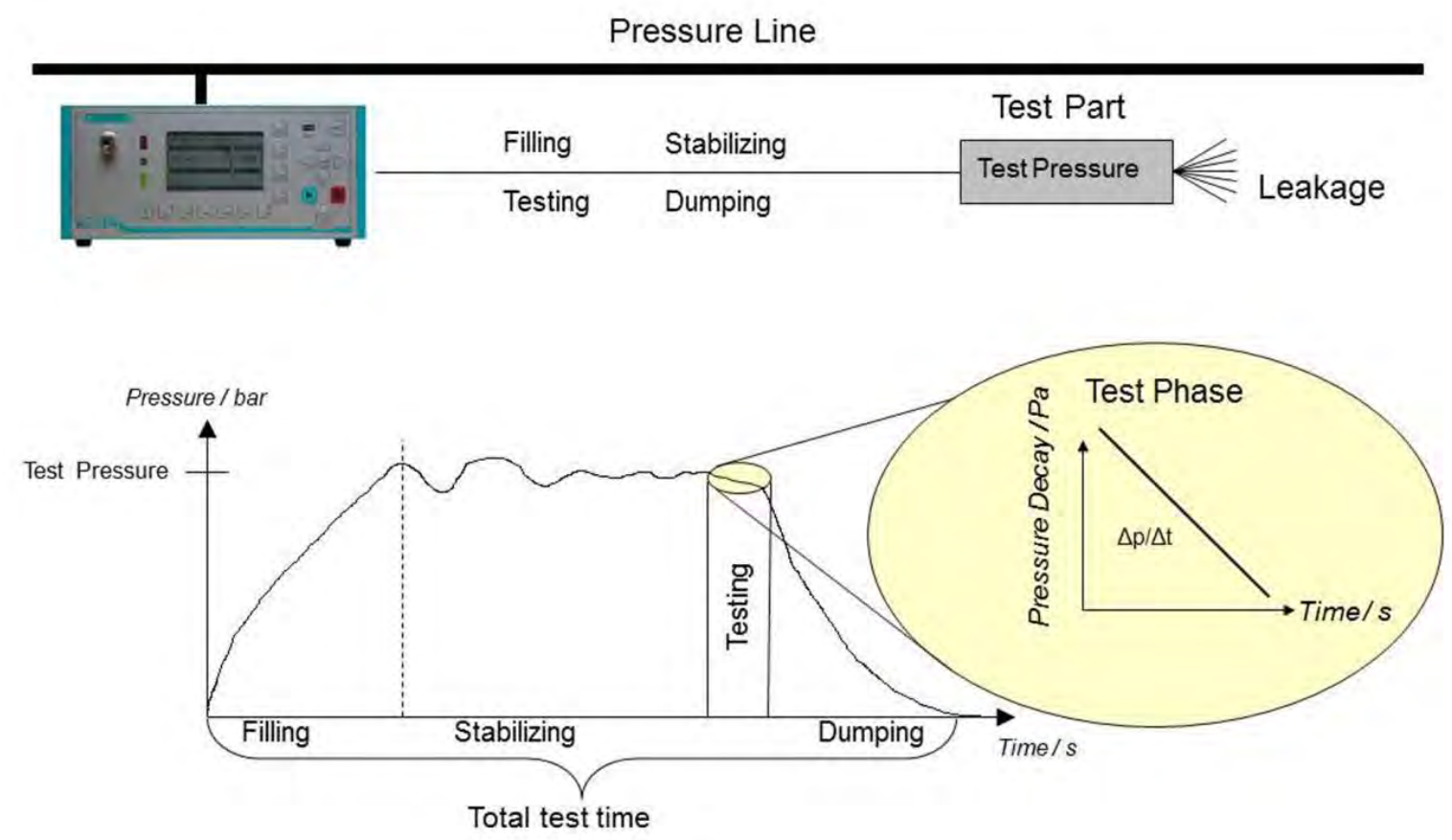

Fig. 1. Principle of the pressure decay test of direct fillable test parts.

\section{Leak Rate Formula}

The expected pressure decay in time in the test phase can be calculated by the use of the socalled leak rate formula. This is an approximation for the leakage induced pressure decay in a stable test regime (eq. (1)):

$$
\frac{\Delta \mathrm{p}}{\Delta \mathrm{t}}\left[\frac{\mathrm{Pa}}{\mathrm{s}}\right]=\frac{\mathrm{Q}_{\mathrm{L}}\left[\mathrm{cm}^{3} / \mathrm{min}\right]}{\mathrm{V}_{\text {eff }}\left[\mathrm{cm}^{3}\right]} \cdot \frac{100.000 \mathrm{~Pa}}{60 \mathrm{~s} / \mathrm{min}}
$$

where

$$
\begin{aligned}
& \Delta \mathrm{p} / \Delta \mathrm{t}=\text { Pressure decay in time } \\
& \mathrm{V}_{\text {eff }} \quad=\text { Effective test volume }
\end{aligned}
$$




\section{Test Method "Closed Components" for encapsulated Test Parts}

Encapsulated test parts, like most of the sensor types, cannot be filled inside with pressurized air. A typical example to demonstrate this test method is an encapsulated photoelectric sensor (see Fig. 2). These types of test parts are placed in a hood which encloses the test part as closely as possible. The hood is pressurized. The temporal pressure decay of the pressure in the test part is measured. This type of test is called "closed component test" or "hood test".

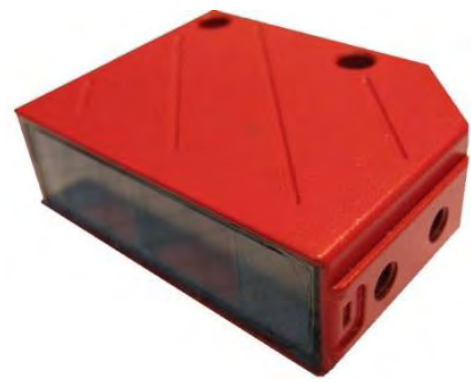

Fig. 2. Photoelectric sensor as an example for a encapsulated test part.
Hereby occurs the following problem: If the test part has a major leak (so-called gross leak), it is already filled with compressed air during the filling phase of the hood. In this case, only the tightness of the hood surrounding the test part would be measured. Therefore it has to be controlled in the first step that the test part has no gross leak (gross leak test). After this the fine leak test is performed by means of a pressure decay measurement (see above).

The gross leak test is done in the following way (see Fig. 3): A reservoir volume, which is integrated in the test device, is filled to a pressure $p_{1}$ and separated from the pressure regulator. Then the check valve of the internal reservoir volume is opened and the air is flooded through the measuring line into the hood. Hereby the pressure decreases to a lower pressure $p_{2}$. The ratio $p_{2} / p_{1}$ is a measure for the volume which is filled. The pressure values are measured with a relative pressure sensor.
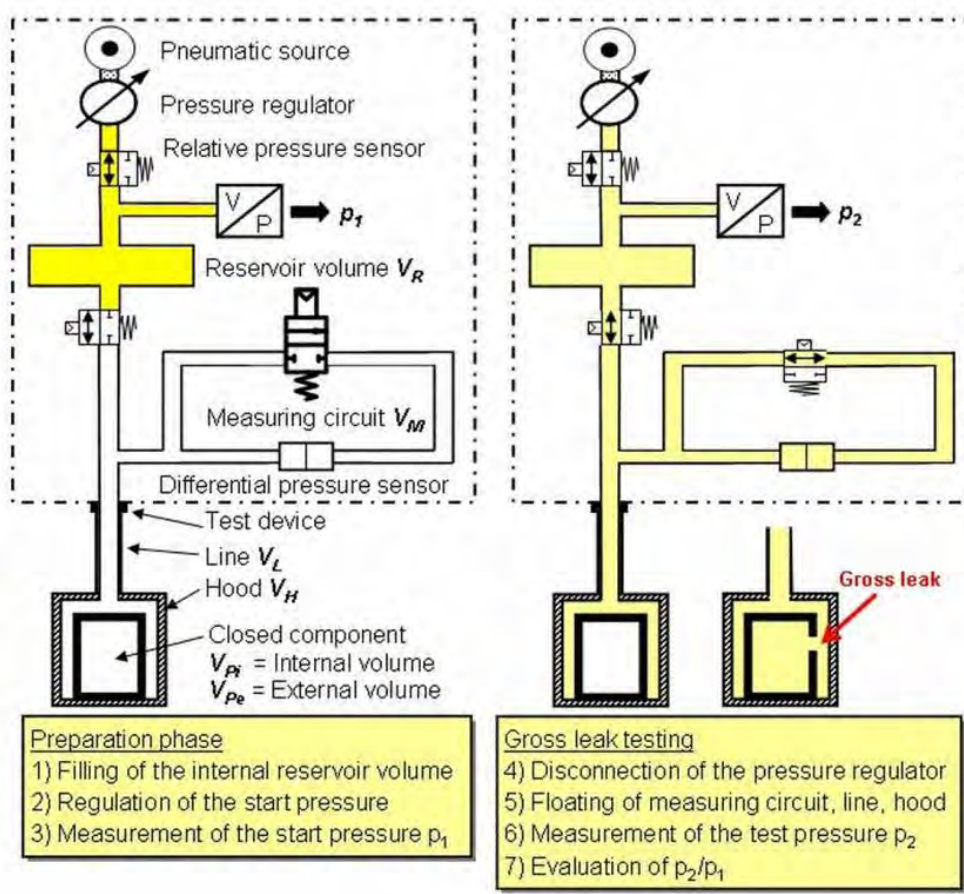

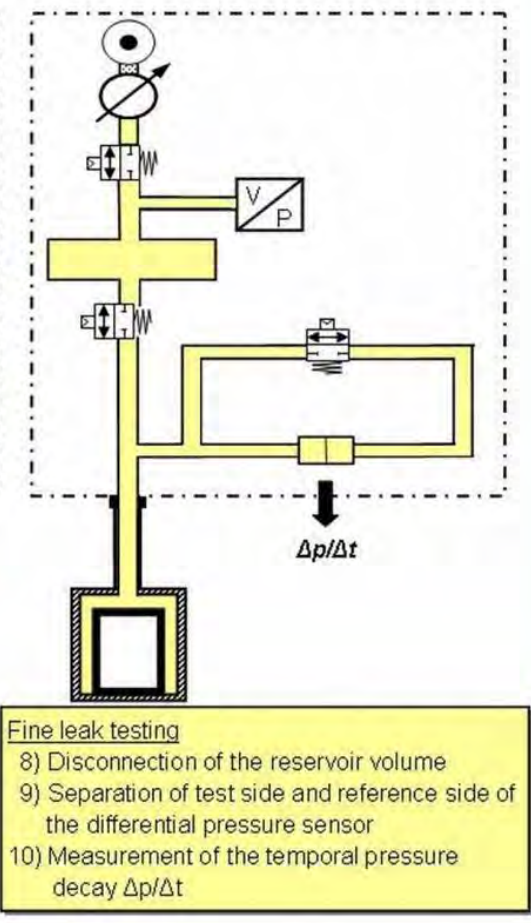

Fig. 3. Principle of the leak test of encapsulated test parts. The total test consists of two steps: After the gross leak test follows the fine leak test.

If the test part has no gross leak, the following applies (eq. (2)):

$$
\frac{p_{2}}{p_{1}}=\frac{V_{R}}{V_{R}+V_{M}+V_{L}+V_{H}-V_{P e}}
$$

In case of a gross leak the internal volume of the test part is filled additionally and the following applies (eq. (3))

$$
\frac{p_{2}}{p_{1}}=\frac{V_{R}}{V_{R}+V_{M}+V_{L}+V_{H}-V_{P e}+V_{P i}}
$$


where

$\mathrm{V}_{\mathrm{R}}=$ Reservoir volume

$\mathrm{V}_{\mathrm{M}}=$ Volume of the measuring circuit

$\mathrm{V}_{\mathrm{L}}=$ Volume of the measuring line

$\mathrm{V}_{\mathrm{H}}=$ Volume of the empty hood

$\mathrm{V}_{\mathrm{Pe}}=$ External volume of the test part

$\mathrm{V}_{\mathrm{Pi}}=$ Internal volume of the test part

(filled in case of a gross leak)

These relationships are a result of the ideal gas equation, assuming constant temperature (isothermal change of state). In the initial state, the volumes are at atmospheric pressure level, so that in these formulas, the pressures are to be used as positive and negative relative pressures.

\section{Example}

The outer volume of a sensor is $5 \mathrm{~cm}^{3}$. The sensor is encapsulated and has an internal volume of $2 \mathrm{~cm}^{3}$, which is filled in case of a gross leak. The hood, surrounding the sensor, has a volume of $10 \mathrm{~cm}^{3}$. The test device has an internal reservoir volume of $45 \mathrm{~cm}^{3}$, the internal measuring circuit has a volume of $5 \mathrm{~cm}^{3}$. The measuring line has a length of $0.5 \mathrm{~m}$ and an inner diameter of $4 \mathrm{~mm}$. This corresponds to a line volume of $6.3 \mathrm{~cm}^{3}$. In this case, the pressure ratio is 0.734 in case of a sensor without gross leak. If the sensor has a major leak, there is a pressure ratio of 0.711 .

In case of a non gross leak sensor the fine leak follows. To perform the fine leak test at a test pressure of $p_{2}=500$ mbar, a positive pressure of $p_{1}=681$ mbar has to be set as the start value.

\section{Technical Features of the Leak Tester}

Fundamental physical effects (temperature adjustment of the compressed air, disturbances in the compressed air) can hardly be influenced, but the test device can be optimized regarding the components which have a sensitive influence upon the test process.

The distinction between a test part with gross leak and a test part without gross leak is more difficult if the fillable internal volume of the test part is quite small compared to the sum of the other volumes (see eq. (1) and (2)).

By the use of a relative pressure sensor for the measurement of the pressures $p_{1}$ and $p_{2}$ a difference of at least about 0.02 between the gross leak pressure ratio and the non-gross leak pressure ratio is needed for a stable leak test process integrated in the production line.
This can be improved by the use of more sensitive pressure sensors (see below).

The resolution and the total test time in the leak test of small-volume test parts can be optimized by the following procedures:

- Use of a measuring line with a small inner diameter, provided this does not hinder the filling process too much. If the measuring line has an inner diameter of $3 \mathrm{~mm}$, the line volume can be reduced by $44 \%$ as an equal length measuring line with $4 \mathrm{~mm}$ inner diameter.

- Use of special internal electrical valves that have an extremely low switching kick. This reduces the time for the relaxation of the air disturbances caused by the switching process.

- Reduction of the internal volumes of the test device (especially measuring circuit volume).

- Use of a hood that encloses the test part as closely as possible. Hereby the test part tolerances have to be taken into account. In practice it is possible, that the hood has just a surrounding distance of only $0.2 \mathrm{~mm}$ to the outer contour of the test part.

- Use of a much more sensitive pressure sensor (differential pressure sensor instead of relative pressure) to detect even finer differences in the pressure ratio $p_{2} / p_{1}$.

\section{Practical Applications - High Speed Pressure Decay Test and Detection of Assembly Defects}

With such an optimized instrument like the leak tester, type CETATEST 515 in the variant "closed components, high resolution" (see Fig. 4 ), the assembly of microparts can be tested, too. The volume which can be filled with air can be taken as a measure to detect assembly defects like a missing o-ring. In a test part, which has a fillable volume of $20 \mathrm{~cm}^{3}$ and which can be filled directly, a volume difference of only $0.03 \mathrm{~cm}^{3}$ can be detected by using a positive relative pressure of 900 mbar This corresponds to the volume of an o-ring of $12 \mathrm{~mm}$ diameter and $1 \mathrm{~mm}$ cross section.

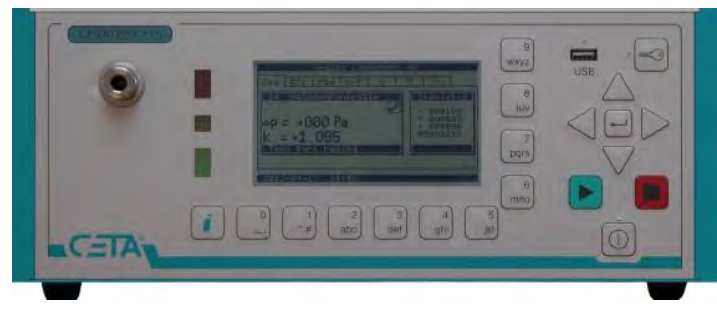

Fig. 4. Leak tester type CETATEST 515 for the leak test of encapsulated test parts with small volume. 
The leak test of encapsulated small volume test parts (external volume approx. 0,5 $\mathrm{cm}^{3}$ ) have been realized in the production line with a total test times in the range of 1 to $2 \mathrm{~s}$. This can result in several million cycles in one year in a production line working to full capacity. The internal components in the test device must be designed accordingly for a long service life.

In the leak test of low-volume special valves by the pressure decay leak test using the CETATEST 515 (in the variant "pressure decay high speed") a total test time of just $750 \mathrm{~ms}$ has been realized. In this very short time a complete leak test cycle consisting of filling, stabilizing, testing and dumping is performed. 